\title{
Search for UHE neutrinos in coincidence with LIGO GW150914 event with the Pierre Auger Observatory
}

\author{
Lili Yang ${ }^{a}$, for the Pierre Auger Collaboration ${ }^{b}$ \\ ${ }^{a}$ Laboratory for Astroparticle Physics, University of Nova Gorica, Slovenia \\ ${ }^{b}$ Observatorio Pierre Auger, Av. San Martin Norte 304, 5613 Malargue, Argentina \\ (Full author list: http://www.auger.org/archive/authors_2016_06.html) \\ email: Lili.Yang@ung.si
}

\begin{abstract}
The first gravitational wave transient GW150914 was observed by Advanced LIGO on September 14th, 2015 at 09:50:45 Universal Time. In addition to follow-up electromagnetic observations, the detection of neutrinos will probe deeply and more on the nature of astrophysical sources, especially in the ultra-high energy regime. Neutrinos in the $\mathrm{EeV}$ energy range were searched in data collected at the surface detector of the Pierre Auger Observatory within \pm $500 \mathrm{~s}$ and 1 day after the GW150914 event. No neutrino candidates were found. Based on this non-observation, we derive the first and only neutrino fluence upper limit at $\mathrm{EeV}$ energies for this event at $90 \% \mathrm{CL}$, and report constraints on existence of accretion disk around mergers.
\end{abstract}

Keywords. gravitational waves, neutrinos, black hole physics

\section{Introduction}

The LIGO collaboration recently announced the first detection of gravitational wave event GW150914, occurring on September 14th, 2015 (Abbott et al. 2016). This event was produced by the inspiral and merger of a pair of black holes lying at a luminosity distance $D=410_{-180}^{+160} \mathrm{Mpc}$, according to the waveform analysis. The merger can result in a black hole, relic magnetic fields and debris remaining from the formation of the black holes, providing the environment to accelerate cosmic rays to ultrahigh energies (Kotera \& Silk 2016). As byproducts, ultrahigh energy gamma rays and neutrinos can also be generated by the hadronic interactions of cosmic rays and ambient surroundings. Even though such a scenario is unlikely with current consensus, the Fermi Gammaray Burst Monitor (GBM) revealed a weak transient with the location consistent with GW150914, and $0.4 \mathrm{~s}$ after (Connaughton et al. 2016). However, no other gamma-ray or $\mathrm{X}$-ray detector found any coincident signal. The debates have been raised about whether this GBM detection is associated with GW150914 (Xiong 2016) and whether the merger of two black holes will produce any electromagnetic emission (Liu \& Zhang 2009).

The discovery of GW150914 and the follow-up detections of GW151226 and LVT 151012 (Abbott et al. 2016) opened a new era for multi-messenger astronomy and motivated neutrino follow-up search at all energies. At PeV energies, it has been performed by ANTARES and IceCube neutrino observatories, and no neutrino candidate was found in both temporal and spatial coincidence during the event period set by (Adrian-Martinez et al. 2016). In the EeV energy ranges, with the surface detector (SD) array, the Pierre Auger Observatory is able to detect and identify ultrahigh energy (UHE) neutrinos at high zenith angles (Abreu et al. 2013). In this work, we present results of an UHE neutrino follow-up search for GW150914 with the SD and put stringent constraints on the source of GW150914 event. 


\section{UHE neutrinos coincidence search}

The placed array covers an area of $3000 \mathrm{~km}^{2}$, consisting of 1660 water-Cherenkov stations in a triangular grid with $1.5 \mathrm{~km}$ spacing (Allekotte et al. 2008). With the SD, neutrino induced air showers can be distinguished from the background of air showers initiated by hadronic cosmic rays at energies above $100 \mathrm{PeV}$. The idea for identification is quite simple, the neutrinos can generate showers deeply in the atmosphere, while nucleons and photons interact shortly after they enter the atmosphere. Therefore, at large zenith angles $\left(\theta>60^{\circ}\right)$, neutrino showers keep a considerable amount of electromagnetic component at the ground ("young" showers), with signals spread in time over hundreds of nano-seconds; on the other hand, at large zenith angles, the atmosphere is thick enough so that electromagnetic component of the nucleonic cosmic ray showers gets absorbed, leaving the muonic components dominated at ground level ("old showers"), with narrower signals spreading over tens of ns. Based on this characteristic of young and old shower front traces, with the 25 ns time resolution of Flash Analog-Digital converters (FADC), neutrino shower signals can be distinguished from background. In this work, two types of neutrino-induced showers are searched for above $100 \mathrm{PeV}$ :

(1). Earth-skimming (ES) showers at zenith angles $90^{\circ} \leqslant \theta \leqslant 95^{\circ}$. They are induced by tau neutrinos $\nu_{\tau}$, which travel in a slightly upward direction with respect to ground, and can skim the Earth's crust and interact near the surface, inducing a tau lepton which escapes the Earth and decays in flight in the atmosphere, close to the SD. Typically, only $\nu_{\tau}$-induced showers with zenith angles $90^{\circ} \leqslant \theta \leqslant 95^{\circ}$ may be identified.

(2). Downward-going high (DGH) zenith angle showers of any flavor moving down at $75^{\circ}$ $\leqslant \theta \leqslant 90^{\circ}$ with respect to the vertical. They are initiated deeply in the atmosphere close to the SD array through charged-current or neutral-current interactions.

The SD array is located at the latitude of $35.2^{\circ}$ (Aab et al. 2015), sensitive to specific portion of the sky at each instant time. During the searching periods of $\pm 500 \mathrm{~s}$ around GW150914 and one day after, the SD was monitored every minute and was in stable operation, with $\sim 97.5 \%$ of stations active. The search window of $\pm 500 \mathrm{~s}$ is an estimated upper bound based on the observation of time duration of GRB prompt emission (Baret et al. 2011), when UHE neutrinos are expected from the interactions of accelerated protons with the observed gamma-rays (Moharan et al. 2016). On the other hand the choice of one day after the event is based on the conservative time bound accounting for the GRB afterglow emissions.

In Fig. 1, the reconstructed $90 \%$ confidence level (CL) probability density contour of GW150914 is shown in equatorial coordinates, and derived from the full parameter estimation of the signal, covering $590 \mathrm{deg}^{2}$. The position is not well constrained (AdrianMartinez et al. 2016). In the same figure, the field of view (FOV) of ES and DGH channels of Auger are also plotted. Here, we assume that within a $1000 \mathrm{~s}$ time interval, the FOVs do not change with time. Within \pm 500 s around GW150914, only a small portion of the event position is overlapping with the FOV of the DGH channel and not overlapping with the ES channel.

Later, we calculated the fractions of one sidereal day of the source at declination $\delta$ visible in the ES and DGH angular ranges. In Fig. 2, the sky map of the GW150914 event at $90 \% \mathrm{CL}$ is shown and the color scales indicate the fractions changing with source direction. For ES and DGH channels, up to $\sim 17 \%$ and $\sim 35 \%$ of this source can be seen with Auger in one day, respectively. These numbers of visible fractions strongly depend on the duration and sky position of the sources, and can be significantly different for various sources. As we can see, the declination of GW150914 visible to Auger is $-1.0^{\circ}$ to $-14.5^{\circ}$ and $-38.5^{\circ}$ to $-78.0^{\circ}$. We applied the neutrino selection criteria which 


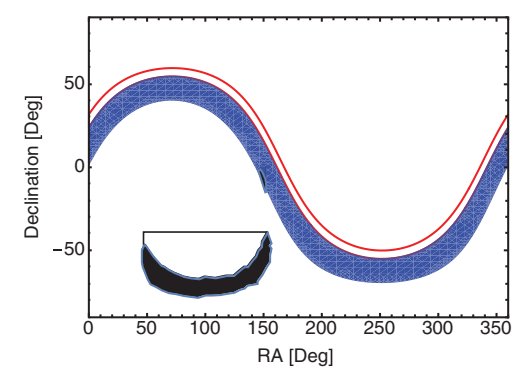

Figure 1: The upper blank (lower shaded) sinusoid bands represent the Auger instant field of view of ES (DGH) channels at the moment of GW150914 transient in equatorial coordinates. The two irregular spots in the lower left corner show the reconstructed probability density contour of GW150914 at 90\% CL.
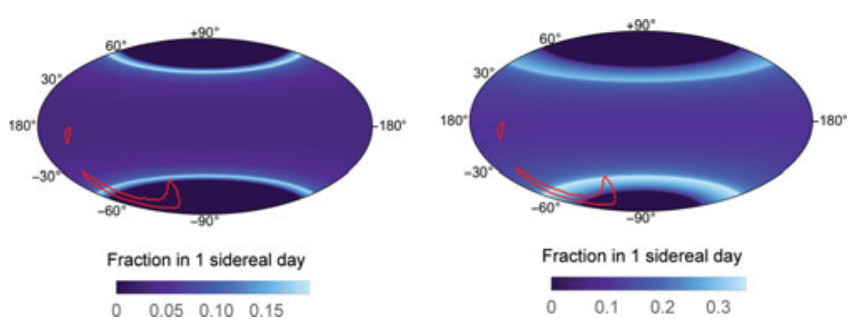

Figure 2: The sky map of the GW150914 event at 90\% CL in solid curves. The color scales indicate the fractions of one sidereal day the source is visible at each declination changing with source direction in ES (left panel) and DGH (right panel) channels

were established as in reference Abreu et al. (2013) and searched for UHE neutrinos for both time windows. No neutrino candidates were found in temporal coincidence with GW150914.

\section{Source constraints}

Since there was no coincident neutrino candidate found, we were allowed to calculate the upper limits to the UHE neutrino spectral fluence as a function of source declination $\delta$. We assumed a standard $\mathrm{d} N / \mathrm{d} E=k E^{-2}$ source model, hence a $90 \%$ CL upper limit on normalization $k$ can be obtained as $k(\delta)=\frac{2.39}{\int E_{\nu}^{-2} \epsilon\left(E_{\nu}, \delta\right) \mathrm{d} E_{\nu}}$, where $\epsilon\left(E_{\nu}, \delta\right)$ is the exposure to UHE neutrinos, which was computed following the reference (Abraham et al. 2008) for ES and DGH channels separately. With this equation, we obtain upper limits to the normalization $k_{\mathrm{ES}}$ and $k_{\mathrm{DGH}}$ for both channels and a combined upper limits to $k$ with the formula $k^{-1}=\left(k_{\mathrm{ES}}\right)^{-1}+\left(k_{\mathrm{DGH}}\right)^{-1}$. Next, the upper limits on the UHE neutrino spectral fluence $E^{-2} \mathrm{~d} N / \mathrm{d} E \times T=k(\delta) \times T$, where $T=1$ day $+500 \mathrm{~s}$ is the search window, are obtained and shown in the left plot in Fig. 3. The shaded bands indicate that the limits are only valid in these declination range at $90 \%$ CL. Fluences above the solid line are excluded at $90 \%$ CL from the non-observation of UHE neutrino events in Auger.

With the assumed neutrino spectrum $\mathrm{d} N / \mathrm{d} E=k E^{-2}$, we integrate the emission flux over energy from $100 \mathrm{PeV}$ to $25 \mathrm{EeV}$ and obtain the constraint $F(\delta)$. Finally, the total emitted energy in neutrinos expressed as $E_{t o t}=F(\delta) \times 4 \pi D^{2}$ is evaluated at three luminosity distances and shown in the right plot of Fig. 3. Energies above each line with respect to the corresponding luminosity distance to the source are excluded at the $90 \%$ CL from the non-observation of UHE neutrinos in Auger. 

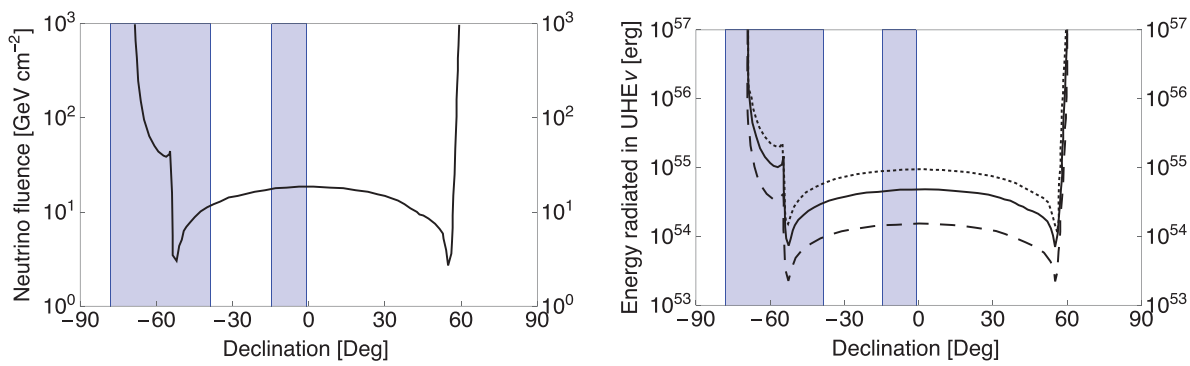

Figure 3: (a) Upper limit on the UHE neutrino spectral fluence per flavor from GW150914 as a function of equatorial declination. The shaded regions indicate the declination ranges which are visible in Auger at 90\% CL. (b) Upper limits on the total energy radiated in UHE neutrinos per flavor from GW150914 as a function of equatorial declination. The dotted line represents the constraints when the source is farther away at $D=410+160$ Mpc, and the dashed line shows when the source is closer to Earth at $D=410-180$ Mpc, corresponding to the $90 \%$ CL interval of possible distances to the source.

\section{Conclusion}

In this work, we present the most stringent upper limit on the neutrino emissions from an identified GW transient in the energies above $100 \mathrm{PeV}$. Considering the energy radiated in gravitational wave from GW150914 is $\sim 5.4 \times 10^{54}$ erg (Abbott et al. 2016), and our most restrictive upper limit on the total energy emitted per flavor in UHE neutrinos achieved at declination $-53^{\circ}$ is $<7.7 \times 10^{53} \mathrm{erg}$, we obtain the upper limit on the fraction of energy radiated in UHE neutrinos relative to the energy emitted in GW150914 is $\sim 14.3 \%$. We have also searched for signals at $\pm 500 \mathrm{~s}$ around and 1 day after GW151226 and LVT151012 and did not find any neutrino candidate, more details given in the paper Aab et al. (2016).

The first detection of GW event has opened a new era for multi-messenger studies involving the joint observations of neutrinos, gamma rays and GWs. This joint analysis will provide strong constraints on the properties of these astrophysical phenomena and provide more accurate directional information. The inferred black hole merger rate is in the range of 9 to $240 \mathrm{Gpc}^{-3} \mathrm{yr}^{-1}$, and more gravitational wave detections are expected in the near future (Abbott et al. 2016).

\section{References}

B. P. Abbott et al., Phys. Rev. Lett. 116, 061102 (2016)

K. Kotera \& J. Silk, Astrophys. J. 823, L29 (2016)

V. Connaughton et al., Astrophys. J. 826, L6 (2016)

S. Xiong, arXiv:1605.05447 [astro-ph.HE]

Y. Liu \& S. N. Zhang, Phys. Lett. B 679, 88 (2009)

B. P. Abbott et al. Phys. Rev. Lett. 116, 241103 (2016)

S. Adrian-Martinez et al., Phys. Rev. D 93, 122010 (2016)

P. Abreu et al., Adv. High Energy Phys. 2013, 708680 (2013)

I. Allekotte et al., Nucl. Instrum. Meth. A 586, 409 (2008)

A. Aab et al., Nucl. Instrum. Meth. A 798, 172 (2015)

B. Baret et al., Astropart. Phys. 35, 1 (2011)

R. Moharana, S. Razzaque, N. Gupta \& P. Meszaros, Phys. Rev. D 93, 123011 (2016)

J. Abraham et al., Phys. Rev. Lett. 100, 211101 (2008)

B. P. Abbott et al., arXiv:1606.04856 [gr-qc].

A. Aab et al., [arXiv:1608.07378 [astro-ph.HE]] 